\title{
Topological Properties of the Complex Vector Lattice of Bounded Measurable Functions
}

\author{
Marian Nowak \\ Faculty of Mathematics, Computer Science and Econometrics, University of Zielona Góra, \\ ul. Szafrana 4A, 65-516 Zielona Góra, Poland \\ Correspondence should be addressed to Marian Nowak; m.nowak@wmie.uz.zgora.pl
}

Received 21 May 2013; Accepted 12 August 2013

Academic Editor: Stanislav Hencl

Copyright (C) 2013 Marian Nowak. This is an open access article distributed under the Creative Commons Attribution License, which permits unrestricted use, distribution, and reproduction in any medium, provided the original work is properly cited.

Let $\Sigma$ be a $\sigma$-algebra of subsets of a nonempty set $\Omega$. Let $B(\Sigma)$ be the complex vector lattice of bounded $\Sigma$-measurable complexvalued functions on $\Omega$ and let $c a(\Sigma)$ be the Banach space of all bounded countably additive complex-valued measures on $\Omega$. We study locally solid topologies on $B(\Sigma)$. In particular, it is shown that the Mackey topology $\tau(B(\Sigma), c a(\Sigma))$ is the finest locally convexsolid $\sigma$-Lebesgue topology on $B(\Sigma)$.

\section{Introduction and Terminology}

For terminology concerning complex vector lattices we refer the reader to [1, Chapter 12, Section 91], [2, Chapter 16], [3]. We denote by $\sigma(L, K)$ and $\tau(L, K)$ the weak topology and the Mackey topology with respect to a dual pair $\langle L, K\rangle$. For a topological vector space $(L, \eta)$ by $(L, \eta)^{\prime}$ or $L_{\eta}^{\prime}$, we denote its topological dual.

We assume that $\Sigma$ is a $\sigma$-algebra of subsets of a nonempty set $\Omega$. Let $B(\Sigma)$ denote the Banach space of all bounded $\sum$-measurable functions $f: \Omega \rightarrow \mathbb{C}$, provided with the uniform norm $\|\cdot\|$. Then, $B(\Sigma)$ with the positive cone $B(\Sigma)^{+}=$ $\{f \in B(\Sigma): f(\omega) \geq 0$ for all $\omega \in \Omega\}$ and a valuation $B(\Sigma) \ni f \mapsto|f| \in B(\Sigma)^{+}$is a complex vector lattice (in the sense of $\left[2\right.$, Section 16-2.2]). By $B_{\mathbb{R}}(\Sigma)$ we denote the Dedekind $\sigma$-complete Banach lattice of all bounded $\Sigma$ measurable functions $u: \Omega \rightarrow \mathbb{R}$, with the natural ordering $u \leq v$ if $u(\omega) \leq v(\omega)$ for all $\omega \in \Omega$ (see [4, Chapter 13.1]). Then, $B(\Sigma)=B_{\mathbb{R}}(\Sigma)+i B_{\mathbb{R}}(\Sigma)$, that is, $B(\Sigma)$ is a complexification of $B_{\mathbb{R}}(\Sigma)$ and $B(\Sigma)$ has the dominated decomposition property, that is, if $|f| \leq u_{1}+u_{2}$, where $f \in B(\Sigma), u_{1}, u_{2} \in B(\Sigma)^{+}$, then there exist $f_{1}, f_{2} \in B(\Sigma)$ such that $f=f_{1}+f_{2}$ and $\left|f_{1}\right| \leq u_{1}$, $\left|f_{2}\right| \leq u_{2}$ (see [1, Theorem 91.3], [3]). This means that $B(\Sigma)$ is a breakable complex vector lattice (see [2, Section 16-3]).

Denote by $b a(\Sigma)$ the Banach space of all bounded finitely additive measures $\mu: \Sigma \rightarrow \mathbb{C}$ with the norm $\|\mu\|=|\mu|(\Omega)$, where $|\mu|(A)$ denotes the variation of $\mu$ on $A \in \Sigma$. It is well known that the Banach dual $B(\Sigma)^{*}$ of $B(\Sigma)$ can be identified with $b a(\Sigma)$ through the integration mapping $b a(\Sigma) \ni \mu \mapsto$ $\Phi_{\mu} \in B(\Sigma)^{*}$, where $\Phi_{\mu}(f)=\int_{\Omega} f d \mu$ for all $f \in B(\Sigma)$ and $\left\|\Phi_{\mu}\right\|=|\mu|(\Omega)$ (see $[5$, Chapter 1, Theorem 13, p.6]. Let $c a(\Sigma)$ stand for the Banach space of all countably additive measures $\mu: \Sigma \rightarrow \mathbb{C}$.

It turns out that the basic notions and results from the theory of vector lattices (in particular, locally solid topologies) can be carried over to the complex vector lattices $B(\Sigma)$ and $b a(\Sigma)$.

A subset $H$ of $B(\Sigma)$ is said to be solid if it follows from $|f| \leq|g|$ and $f \in B(\Sigma), g \in H$ that $f \in H$. Every subset $H$ of $B(\Sigma)$ is included in the smallest (with respect to the inclusion) solid set, called the solid hull of $H$ and denoted by $\operatorname{sol}(H)$. Clearly,

$$
\operatorname{sol}(H)=\{g \in B(\Sigma):|g| \leq|f| \text { for some } f \in H\} \text {. }
$$

A linear Hausdorff topology $\tau$ on $B(\Sigma)$ is said to be locally solid if it has a local base at 0 consisting of solid sets in $B(\Sigma)$.

In Section 2, we study locally solid topologies on $B(\Sigma)$. In Section 3, we develop the duality theory of locally convexsolid topologies on $B(\Sigma)$. In Section 4 , it is shown that the natural Mackey topology $\tau(B(\Sigma), c a(\Sigma))$ is the finest locally convex-solid $\sigma$-Lebesgue topology on $B(\Sigma)$ (see Theorem 16 below). 


\section{Locally Solid Topologies on $B(\Sigma)$}

Using the dominated decomposition property for $B(\Sigma)$ we can easily obtain the following result (see [6, Theorem 1.11]).

Proposition 1. The convex hull conv $H$ of a solid subset $H$ of $B(\Sigma)$ is solid.

Proposition 2. Let $\tau$ be a locally solid topology on $B(\Sigma)$. Then, the $\tau$-closure $c_{\tau} H$ of a solid subset $H$ of $B(\Sigma)$ is solid.

Proof. Let $\mathscr{B}_{\tau}$ be a local base at 0 for $\tau$ consisting of solid sets. Then $\mathrm{cl}_{\tau} H=\bigcap\left\{H+V: V \in \mathscr{B}_{\tau}\right\}$. Assume that $|f| \leq|g|$, where $f \in B(\Sigma), g \in \mathrm{cl}_{\tau} H$, and let $V_{0} \in \mathscr{B}_{\tau}$. Then, $g=g_{1}+g_{2}$, where $g_{1} \in H$ and $g_{2} \in V_{0}$. Since $|f| \leq|g| \leq\left|g_{1}\right|+\left|g_{2}\right|$ (by the dominated decomposition property for $B(\Sigma)$ ), there exist $f_{1}, f_{2} \in B(\Sigma)$ such that $f=f_{1}+f_{2}$ and $\left|f_{i}\right| \leq\left|g_{i}\right|$ for $i=1,2$. Hence, $f_{1} \in H$ and $f_{2} \in V_{0}$ because both sets $H$ and $V$ are solid. Thus, $f \in H+V_{0}$ for every $V_{0} \in \mathscr{B}_{\tau}$, so $f \in \mathrm{cl}_{\tau} H$. This means that $\mathrm{cl}_{\tau} H$ is solid, as desired.

Following the notion of a modulus of an operator between vector lattices (see [7, Definition 1.8 and Theorem 1.10]), for a linear functional $\Phi: B(\Sigma) \rightarrow \mathbb{C}$ let us put

$$
|\Phi|(u):=\sup \{|\Phi(h)|: h \in B(\Sigma),|h| \leq u\}
$$

for all $u \in B(\Sigma)^{+}$.

Proposition 3. For a linear functional $\Phi$ on $B(\Sigma)$ the following statements are equivalent:

(i) $\Phi \in B(\Sigma)^{*}$.

(ii) $|\Phi|(u)<\infty$ for all $u \in B(\Sigma)^{+}$.

Proof. (i) $\Rightarrow$ (ii) By way of contradiction, assume that there exists $\Phi_{0} \in B(\Sigma)^{*}$ such that $\left|\Phi_{0}\right|\left(u_{0}\right)=\infty$ for some $u_{0} \in$ $B(\Sigma)^{+}$. Hence, there exists a sequence $\left(h_{n}\right)$ in $B(\Sigma)$ such that $\left|h_{n}\right| \leq u_{0}$ and $\left|\Phi_{0}\left(h_{n}\right)\right| \geq n$ for $n \in \mathbb{N}$. Since $\left\|(1 / n) h_{n}\right\| \rightarrow 0$, we get $(1 / n)\left|\Phi_{0}\left(h_{n}\right)\right| \rightarrow 0$, which is in contradiction with $\left|\Phi_{0}\left(h_{n}\right)\right| \geq n$.

(ii) $\Rightarrow$ (i) Assume that there exists a linear functional $\Phi_{0}$ on $B(\Sigma)$ such that $\left|\Phi_{0}\right|(u)<\infty$ for all $u \in B(\Sigma)^{+}$and $\Phi_{0} \notin B(\Sigma)^{*}$. Then, there exists a sequence $\left(f_{n}\right)$ in $B(\Sigma)$ such that $\left\|f_{n}\right\|=$ 1 and $\left|\Phi_{0}\left(f_{n}\right)\right|>n^{3}$ for all $n \in \mathbb{N}$. Since $\sum_{n=1}^{\infty}\left(1 / n^{2}\right)\left\|f_{n}\right\|<$ $\infty$ and the space $(B(\Sigma),\|\cdot\|)$ is complete, there exists $u_{0} \in$ $B(\Sigma)^{+}$such that $\sum_{n=1}^{\infty}\left(1 / n^{2}\right)\left|f_{n}\right|=u_{0}$. Then, $\left(1 / n^{2}\right)\left|f_{n}\right| \leq u_{0}$, and; hence, $n<\left|\Phi_{0}\left(\left(1 / n^{2}\right) f_{n}\right)\right|<\infty$ for all $n \in \mathbb{N}$, which is impossible.

Thus, the proof is complete.

For $\Phi_{1}, \Phi_{2} \in B(\Sigma)^{*}$ we will write $\left|\Phi_{1}\right| \leq\left|\Phi_{2}\right|$ whenever $\left|\Phi_{1}\right|(u) \leq\left|\Phi_{2}\right|(u)$ for all $u \in B(\Sigma)^{+}$. A subset $\mathscr{K}$ of $B(\Sigma)^{*}$ is said to be solid if $\left|\Phi_{1}\right| \leq\left|\Phi_{2}\right|$, where $\Phi_{1} \in B(\Sigma)^{*}, \Phi_{2} \in \mathscr{K}$ implies that $\Phi_{1} \in \mathscr{K}$. A linear subspace $I$ of $B(\Sigma)^{*}$ is said to be an ideal if $I$ is a solid set in $B(\Sigma)^{*}$.

Note that, if $\left|\Phi_{1}\right| \leq\left|\Phi_{2}\right|$, then $\left\|\Phi_{1}\right\| \leq\left\|\Phi_{2}\right\|$.

Proposition 4. Let $\tau$ be a locally solid topology on $B(\Sigma)$. Then, $(B(\Sigma), \tau)^{\prime}$ is an ideal of $B(\Sigma)^{*}$.
Proof. To show that $(B(\Sigma), \tau)^{\prime} \subset B(\Sigma)^{*}$, by the way of contradiction, assume that for some $\Phi_{0} \in(B(\Sigma), \tau)^{\prime}$ we have $\Phi_{0} \notin B(\Sigma)^{*}$, so in view of Proposition 3, we obtain that $\left|\Phi_{0}\right|\left(u_{0}\right)=\infty$ for some $u_{0} \in B(\Sigma)^{+}$. Hence, there exists a sequence $\left(h_{n}\right)$ in $B(\Sigma)$ such that $\left|h_{n}\right| \leq u_{0}$ and $\left|\Phi_{0}\left(h_{n}\right)\right| \geq n$ for all $n \in \mathbb{N}$. Since $(1 / n) u_{0} \rightarrow 0$ for $\tau$ and $\tau$ is locally solid, we get $(1 / n) h_{n} \rightarrow 0$ for $\tau$. Hence, $\Phi_{0}\left((1 / n) h_{n}\right) \rightarrow 0$, which is in contradiction with $\left|\Phi_{0}\left(h_{n}\right)\right| \geq n$ for all $n \in \mathbb{N}$.

To see that $(B(\Sigma), \tau)^{\prime}$ is an ideal of $B(\Sigma)^{*}$, assume that $\left|\Phi_{1}\right| \leq\left|\Phi_{2}\right|$, where $\Phi_{1} \in B(\Sigma)^{*}$ and $\Phi_{2} \in(B(\Sigma), \tau)^{\prime}$. Let $f_{\alpha} \rightarrow 0$ for $\tau$ and $\varepsilon>0$ be given. Then, there exists a net $\left(h_{\alpha}\right)$ in $B(\Sigma)$ such that $\left|h_{\alpha}\right| \leq\left|f_{\alpha}\right|$ for each $\alpha$ and $\left|\Phi_{2}\right|\left(\left|f_{\alpha}\right|\right) \leq$ $\left|\Phi_{2}\left(h_{\alpha}\right)\right|+\varepsilon$. Clearly, $h_{\alpha} \rightarrow 0$ for $\tau$ because $\tau$ is locally solid, so $\Phi_{2}\left(h_{\alpha}\right) \rightarrow 0$. Since $\left|\Phi_{1}\left(f_{\alpha}\right)\right| \leq\left|\Phi_{1}\right|\left(\left|f_{\alpha}\right|\right) \leq\left|\Phi_{2}\right|\left(\left|f_{\alpha}\right|\right) \leq$ $\left|\Phi_{2}\left(h_{\alpha}\right)\right|+\varepsilon$, we get $\Phi_{1}\left(f_{\alpha}\right) \rightarrow 0$, so $\Phi_{1} \in(B(\Sigma), \tau)^{\prime}$, as desired.

\section{Duality of the Space $B(\Sigma)$}

A locally convex Hausdorff topology $\tau$ on $B(\Sigma)$ is said to be locally convex-solid if it has a local base at 0 consisting of convex and solid sets in $B(\Sigma)$. In view of Propositions 1 and 2 , we see that for a locally convex-solid topology $\tau$ on $B(\Sigma)$ the collection of all $\tau$-closed convex and solid $\tau$-neighborhoods of 0 forms a local base at 0 for $\tau$.

In this section, we develop the duality theory of locally convex-solid topologies on $B(\Sigma)$. We start with the following lemma.

Lemma 5. Let $\mu \in b a(\Sigma)$. Then, for each $u \in B(\Sigma)^{+}$we have

$$
\begin{aligned}
\left|\Phi_{\mu}\right|(u) & =\int_{\Omega} u d|\mu| \\
& =\sup \left\{\left|\int_{\Omega} h d \mu\right|: h \in B(\Sigma),|h| \leq u\right\} .
\end{aligned}
$$

In particular, we have for $A \in \Sigma$

$$
|\mu|(A)=\sup \left\{\left|\int_{A} h d \mu\right|: h \in B(\Sigma),\|h\| \leq 1\right\} .
$$

Proof. Let $u \in B(\Sigma)^{+}$and $h \in B(\Sigma)$ with $|h| \leq u$. Then, we have

$$
\left|\int_{\Omega} h d \mu\right| \leq \int_{\Omega}|h| d|\mu| \leq \int_{\Omega} u d|\mu|
$$

so $\left|\Phi_{\mu}\right|(u) \leq \int_{\Omega} u d|\mu|$. Now we shall show that $\int_{\Omega} u d|\mu| \leq$ $\left|\Phi_{\mu}\right|(u)$. Indeed, let $\varepsilon>0$ be given. Then, there exists a $\Sigma$ simple function $s=\sum_{i=1}^{k} \alpha_{i} \mathbb{1}_{A_{i}}$ such that $\alpha_{i}>0, s \leq u$ and

$$
\left|\int_{\Omega} u d\right| \mu\left|-\sum_{i=1}^{k} \alpha_{i}\right| \mu\left|\left(A_{i}\right)\right| \leq \frac{\varepsilon}{2} .
$$

Let $\alpha=\sum_{i=1}^{k} \alpha_{i}$. Then for each $i=1,2, \ldots, k$ one can choose a finite $\sum$-partition $\left\{A_{i j}: j=1, \ldots, k_{i}\right\}$ of $A_{i}$ and $\varepsilon_{i j} \in \mathbb{C}$ with $\left|\varepsilon_{i j}\right|=1, \varepsilon_{i j} \mu\left(A_{i j}\right)=\left|\mu\left(A_{i j}\right)\right|$ for $j=1, \ldots, k_{i}$ such that

$$
|\mu|\left(A_{i}\right) \leq \sum_{i=1}^{k_{i}} \varepsilon_{i j} \mu\left(A_{i j}\right)+\frac{\varepsilon}{2 \alpha} .
$$


Let $s_{0}=\sum_{i=1}^{k} \sum_{j=1}^{k_{i}} \alpha_{i} \varepsilon_{i j} \mathbb{1}_{A_{i j}}$. Then $\left|s_{0}\right|=s \leq u$ and we have

$$
\begin{aligned}
\left|\int_{\Omega} u d\right| \mu\left|-\int_{\Omega} s_{0} d \mu\right| \\
\leq\left|\int_{\Omega} u d\right| \mu\left|-\sum_{i=1}^{k} \alpha_{i}\right| \mu\left|\left(A_{i}\right)\right| \\
\quad+\left|\sum_{i=1}^{k} \alpha_{i}\right| \mu\left|\left(A_{i}\right)-\sum_{i=1}^{k} \sum_{j=1}^{k_{i}} \alpha_{i} \varepsilon_{i j} \mu\left(A_{i j}\right)\right| \\
\leq \frac{\varepsilon}{2}+\sum_{i=1}^{k} \alpha_{i}|| \mu\left|\left(A_{i}\right)-\sum_{j=1}^{k_{i}} \varepsilon_{i j} \mu\left(A_{i j}\right)\right| \\
\leq \frac{\varepsilon}{2}+\sum_{i=1}^{k} \alpha_{i} \frac{\varepsilon}{2 \alpha}=\frac{\varepsilon}{2}+\frac{\varepsilon}{2}=\varepsilon .
\end{aligned}
$$

Hence, $\int_{\Omega} u d|\mu| \leq\left|\int_{\Omega} s_{0} d \mu\right|+\varepsilon$, and it follows that $\int_{\Omega} u d|\mu| \leq$ $\left|\Phi_{\mu}\right|(u)$.

Since $\left|\Phi_{\mu}\right|: B(\Sigma)^{+} \rightarrow \mathbb{R}^{+}$is additive, $\left|\Phi_{\mu}\right|$ can be uniquely extended to a positive linear functional (denoted by $\left|\Phi_{\mu}\right|$ again) $\left|\Phi_{\mu}\right|: B(\Sigma) \rightarrow \mathbb{C}$ (see [2, Theorem 16-4.7]). Then, for $f \in B(\Sigma)$ we have

$$
\begin{aligned}
\left|\Phi_{\mu}\right|(f)= & \left|\Phi_{\mu}\right|\left((\operatorname{Re} f)^{+}\right)-\left|\Phi_{\mu}\right|\left((\operatorname{Re} f)^{-}\right) \\
& +i\left|\Phi_{\mu}\right|\left((\operatorname{Im} f)^{+}\right)-i\left|\Phi_{\mu}\right|\left((\operatorname{Im} f)^{-}\right) \\
= & \int_{\Omega}(\operatorname{Re} f)^{+} d|\mu|-\int_{\Omega}(\operatorname{Re} f)^{-} d|\mu| \\
& +i \int_{\Omega}(\operatorname{Im} f)^{+} d|\mu|-i \int_{\Omega}(\operatorname{Im} f)^{-} d|\mu| \\
= & \int_{\Omega} f d|\mu|=\Phi_{|\mu|}(f) .
\end{aligned}
$$

Hence, $\left|\Phi_{\mu}\right|=\Phi_{|\mu|}$.

Proposition 6. For $\mu, \nu \in b a(\Sigma)$ the following statements are equivalent:

(i) $|\mu| \leq|\nu|$.

(ii) $\left|\Phi_{\mu}\right| \leq\left|\Phi_{\nu}\right|$.

Proof. (i) $\Rightarrow$ (ii) Assume that $|\mu| \leq|\nu|$. Then, by Lemma 5, for $u \in B(\Sigma)^{+},\left|\Phi_{\mu}\right|(u)=\int_{\Omega} u d|\mu| \leq \int_{\Omega} u d|\nu|=\left|\Phi_{\nu}\right|(u)$, that is, $\left|\Phi_{\mu}\right| \leq\left|\Phi_{\nu}\right|$.

(ii) $\Rightarrow$ (i) Assume that $\left|\Phi_{\mu}\right| \leq\left|\Phi_{\gamma}\right|$. Then, for $A \in \Sigma$ we have $|\mu|(A)=\left|\Phi_{\mu}\right|\left(\mathbb{1}_{A}\right) \leq\left|\Phi_{\gamma}\right|\left(\mathbb{1}_{A}\right)=|\nu|(A)$.

Now we consider the concept of solidness in the space $b a(\Sigma)$. A subset $\mathscr{M}$ of $b a(\Sigma)$ is said to be solid if $|\nu| \leq|\mu|$, where $v \in b a(\Sigma), \mu \in \mathscr{M}$ implies that $\nu \in \mathscr{M}$.

For a subset $\mathscr{M}$ of $b a(\Sigma)$ let

$\operatorname{sol}(\mathscr{M})=\{\nu \in b a(\Sigma):|\nu| \leq|\mu|$ for some $\mu \in \mathscr{M}\}$.
Then, $\operatorname{sol}(\mathscr{M})$ is the solid hull of $\mathscr{M}$, that is, $\operatorname{sol}(\mathscr{M})$ is the smallest solid set in $\mathscr{M}$ that contains $\mathscr{M}$.

A linear subspace $I$ of $b a(\Sigma)$ is called an ideal in $b a(\Sigma)$ if $I$ is solid. Note that $c a(\Sigma)$ is an ideal of $b a(\Sigma)$. In view of Proposition 6, we see that a subset $\mathscr{M}$ of $b a(\Sigma)$ is solid if and only if the set $\left\{\Phi_{\mu}: \mu \in \mathscr{M}\right\}$ is solid in $B(\Sigma)^{*}$.

Corollary 7. Let $\mu \in b a(\Sigma)$. Then, for $f \in B(\Sigma)$ we have

$$
\begin{aligned}
\left|\Phi_{\mu}\right|(|f|) & =\int_{\Omega}|f| d|\mu| \\
& =\sup \left\{\left|\int_{\Omega} f d \nu\right|: \nu \in b a(\Sigma),|\nu| \leq|\mu|\right\}
\end{aligned}
$$

Moreover, for a subset $\mathscr{M}$ of ba $(\Sigma)$ and each $f \in B(\Sigma)$ we have

$$
\begin{aligned}
& \sup \left\{\left|\Phi_{\mu}\right|(|f|): \mu \in \mathscr{M}\right\} \\
& =\sup \left\{\int_{\Omega}|f| d|\mu|: \mu \in \mathscr{M}\right\} \\
& =\sup \left\{\left|\int_{\Omega} f d \nu\right|: \nu \in \operatorname{sol}(\mathscr{M})\right\} \\
& =\sup \left\{\left|\int_{\Omega} f d \nu\right|: \nu \in \operatorname{conv}(\operatorname{sol}(\mathscr{M}))\right\} .
\end{aligned}
$$

Proof. In view of Proposition 6 and [2, Theorem 16-5.6] we get

$$
\begin{aligned}
\left|\Phi_{\mu}\right|(|f|) & =\sup \left\{\left|\int_{\Omega} f d \nu\right|: \nu \in b a(\Sigma),\left|\Phi_{\nu}\right| \leq\left|\Phi_{\mu}\right|\right\} \\
& =\sup \left\{\left|\int_{\Omega} f d \nu\right|: \nu \in b a(\Sigma),|\nu| \leq|\mu|\right\} .
\end{aligned}
$$

Next, we shall show that for each $f \in B(\Sigma)$,

$$
\sup \left\{\left|\Phi_{\mu}\right|(|f|): \mu \in \mathscr{M}\right\} \leq \sup \left\{\left|\int_{\Omega} f d \nu\right|: \nu \in \operatorname{sol}(\mathscr{M})\right\}
$$

Indeed, let $f \in B(\Sigma)$ be given. Then, for $\mu \in \mathscr{M}$ and $\varepsilon>0$ by $(*)$ there exists $\nu \in b a(\Sigma)$ with $|\nu| \leq|\mu|$ and such that $\left|\Phi_{\mu}\right|(|f|) \leq\left|\int_{\Omega} f d \nu\right|+\varepsilon$. Since $\nu \in \operatorname{sol}(\mathscr{M})$, we get $\left|\Phi_{\mu}\right|(|f|) \leq$ $\sup \left\{\left|\int_{\Omega} f d \nu\right|: \nu \in \operatorname{sol}(\mathscr{M})\right\}+\varepsilon$, and it follows that (12) holds.

It is enough to show that for each $f \in B(\Sigma)$ we have

$$
\begin{aligned}
& \sup \left\{\left|\int_{\Omega} f d \nu\right|: \nu \in \operatorname{conv}(\operatorname{sol}(\mathscr{M}))\right\} \\
& \leq \sup \left\{\left|\Phi_{\mu}\right|(|f|): \mu \in \mathscr{M}\right\} .
\end{aligned}
$$


Indeed, let $v \in \operatorname{conv}(\operatorname{sol}(\mathscr{M}))$. Then, $v=\sum_{i=1}^{n} \alpha_{i} \nu_{i}$ with $\nu_{i} \in$ $\operatorname{sol}(\mathscr{M}), \alpha_{i} \geq 0, i=1, \ldots, n$ and $\sum_{i=1}^{n} \alpha_{i}=1$. Hence, $\left|\nu_{i}\right| \leq\left|\mu_{i}\right|$ for some $\mu_{i} \in \mathscr{M}, i=1,2, \ldots, n$. Hence, for $f \in B(\Sigma)$ we get

$$
\begin{aligned}
\left|\int_{\Omega} f d \nu\right| & \leq \int_{\Omega}|f| d|\nu| \\
& \leq \sum_{i=1}^{n} \alpha_{i} \int_{\Omega}|f| d\left|\nu_{i}\right| \leq \sum_{i=1}^{n} \alpha_{i} \int_{\Omega}|f| d\left|\mu_{i}\right| \\
& \leq\left(\sum_{i=1}^{n} \alpha_{i}\right) \sup \left\{\int_{\Omega}|f| d|\mu|: \mu \in \mathscr{M}\right\} \\
& =\sup \left\{\left|\Phi_{\mu}\right|(|f|): \mu \in \mathscr{M}\right\} .
\end{aligned}
$$

It follows that (13) holds, as desired.

Let $I$ be an ideal of $b a(\Sigma)$ separating the points of $B(\Sigma)$. Then, the pair $\langle B(\Sigma), I\rangle$, under its natural duality

$$
\langle f, \mu\rangle:=\int_{\Omega} f d \mu \quad \text { for } f \in B(\Sigma), \mu \in I
$$

will be referred to as a solid dual system (see [7, Definition 11.7]). For subsets $H$ of $B(\Sigma)$ and $\mathscr{M}$ of $I$, let us put

$$
\begin{gathered}
H^{0}=\left\{\mu \in I:\left|\int_{\Omega} f d \mu\right| \leq 1 \forall f \in H\right\}, \\
{ }^{0}=\left\{f \in B(\Sigma):\left|\int_{\Omega} f d \mu\right| \leq 1 \forall \mu \in \mathscr{M}\right\} .
\end{gathered}
$$

Proposition 8. Let $\langle B(\Sigma), I\rangle$ be a solid dual system.

(i) If $H$ is a solid subset of $B(\Sigma)$, then $H^{0}$ is a solid subset of $I$.

(ii) If $\mathscr{M}$ is a solid subset of $I$, then ${ }^{0} \mathscr{M}$ is a solid subset of $B(\Sigma)$.

Proof. (i) Let $|\nu| \leq|\mu|$ with $\nu \in I$ and $\mu \in H^{0}$. Assume that $f \in$ $H$ and let $h \in B(\Sigma)$ with $|h| \leq|f|$. Then, $h \in H$ because $H$ is solid, so $\left|\int_{\Omega} h d \mu\right| \leq 1$. Hence $\int_{\Omega}|f| d|\mu|=\left|\Phi_{\mu}\right|(|f|) \mid \leq 1$ (see Lemma 5). Thus $\left|\int_{\Omega} f d \nu\right| \leq \int_{\Omega}|f| d|\nu| \leq \int_{\Omega}|f| d|\mu| \leq 1$, that is, $v \in H^{0}$. This means that $H^{0}$ is a solid subset of $I$.

(ii) Let $|f| \leq|g|$, where $f \in B(\Sigma)$ and $g \in{ }^{0} \mathscr{M}$. Since $\mathscr{M}$ is a solid set in $b a(\Sigma)$, by Corollary 7 for $\mu \in \mathscr{M}$, we have

$$
\int_{\Omega}|g| d|\mu|=\sup \left\{\left|\int_{\Omega} g d v\right|: \nu \in \mathscr{M},|\nu| \leq|\mu|\right\} .
$$

Then, for every $\nu \in \mathscr{M}$ with $|\nu| \leq|\mu|$ we have $\left|\int_{\Omega} g d \nu\right| \leq 1$, so $\int_{\Omega}|g| d|\mu| \leq 1$. It follows that

$$
\left|\int_{\Omega} f d \mu\right| \leq \int_{\Omega}|f| d|\mu| \leq \int_{\Omega}|g| d|\mu| \leq 1,
$$

that is, $f \in{ }^{0} \mathscr{M}$.
Observe that for each $\mu \in b a(\Sigma)$, by Corollary 7 the functional $\varrho_{\mu}: B(\Sigma) \rightarrow[0, \infty)$ defined by

$$
\begin{aligned}
\varrho_{\mu}(f) & =\left|\Phi_{\mu}\right|(|f|)=\sup \left\{\left|\int_{\Omega} h d \mu\right|: h \in B(\Sigma),|h| \leq|f|\right\} \\
& =\sup \left\{\left|\int_{\Omega} f d \nu\right|: \nu \in b a(\Sigma),|\nu| \leq|\mu|\right\},
\end{aligned}
$$

is a solid seminorm on $B(\Sigma)$, that is, $\varrho_{\mu}\left(f_{1}\right) \leq \varrho_{\mu}\left(f_{2}\right)$ if $\left|f_{1}\right| \leq$ $\left|f_{2}\right|$.

Let $I$ be an ideal of $b a(\Sigma)$ separating the points of $B(\Sigma)$. The absolute weak topology $|\sigma|(B(\Sigma), I)$ generated by $I$ on $B(\Sigma)$ is the locally convex-solid topology on $B(\Sigma)$ generated by the family of solid seminorms $\left\{\varrho_{\mu}: \mu \in I\right\}$. Note that $|\sigma|(B(\Sigma), I)$ is the topology of uniform convergence on all sets $I_{\mu}=\{\nu \in I:|\nu| \leq|\mu|\}$, where $\mu \in I$.

Proposition 9. For each $\mu \in I$, the set $I_{\mu}$ is convex, solid, and $\sigma(I, B(\Sigma))$-compact in $I$.

Proof. Clearly, $I_{\mu}$ is a convex and solid subset of $I$. We shall show that $I_{\mu}$ is $\sigma(b a(\Sigma), B(\Sigma))$-closed subset of $b a(\Sigma)$. To this end, assume that $\left(\nu_{\alpha}\right)$ is a net in $I_{\mu}$ such that $\int_{\Omega} f d \nu_{\alpha} \rightarrow$ $\int_{\Omega} f d \nu$ for all $f \in B(\Sigma)$, where $\nu \in b a(\Sigma)$. Then

$$
\begin{aligned}
& \left|\int_{\Omega} f d \nu_{\alpha}\right| \longrightarrow\left|\int_{\Omega} f d \nu\right|, \\
& \left|\int_{\Omega} f d \nu_{\alpha}\right| \leq \int_{\Omega}|f| d\left|\nu_{\alpha}\right| \leq \int_{\Omega}|f| d|\mu| .
\end{aligned}
$$

Hence, for each $A \in \Sigma$, we get $\left|\int_{A} f d \nu\right| \leq \int_{A}|f| d|\mu|$ for all $f \in B(\Sigma)$, and by Lemma 5 ,

$$
\begin{aligned}
|\nu|(A) & =\sup \left\{\left|\int_{A} f d \nu\right|: f \in B(\Sigma),\|f\| \leq 1\right\} \\
& \leq \int_{A} \mathbb{1}_{\Omega} d|\mu|=|\mu|(A),
\end{aligned}
$$

that is, $|\nu| \leq|\mu|$. Thus, $\nu \in I_{\mu}$, as desired.

In view of the Banach-Alaoglu theorem, $I_{\mu}$ is a relatively $\sigma(b a(\Sigma), B(\Sigma))$-compact subset of $b a(\Sigma)$. Since $I_{\mu}$ is $\sigma(b a(\Sigma), B(\Sigma))$-closed in $b a(\Sigma), I_{\mu}$ is $\sigma(b a(\Sigma), B(\Sigma))$-compact, and it follows that $I_{\mu}$ is $\sigma(I, B(\Sigma))$-compact.

We define the absolute Mackey topology $|\tau|(B(\Sigma), I)$ as the topology of uniform convergence on all convex, solid and $\sigma(I, B(\Sigma))$-compact subsets of $b a(\Sigma)$. By Proposition 8 $|\tau|(B(\Sigma), I)$ is a locally convex-solid topology on $B(\Sigma)$. Thus, in view of Proposition 9 we have

$$
\begin{aligned}
\sigma(B(\Sigma), I) & \subset|\sigma|(B(\Sigma), I) \\
& \subset|\tau|(B(\Sigma), I) \subset \tau(B(\Sigma), I) .
\end{aligned}
$$

As a consequence, we obtain the following Mackey-Arens type theorem for locally convex-solid topologies on $B(\Sigma)$.

Proposition 10. Let $\tau$ be a locally convex-solid topology on $B(\Sigma)$. Then, $|\sigma|\left(B(\Sigma), B(\Sigma)_{\tau}^{\prime}\right) \subset \tau \subset|\tau|\left(B(\Sigma), B(\Sigma)_{\tau}^{\prime}\right)$. 
Proof. Note that the family $\mathscr{B}_{\tau}$ of all convex, solid and $\tau$ closed $\tau$-neighborhoods of 0 forms a local base at 0 for $\tau$. Then, $\tau$ is the topology of uniform convergence on the family $\left\{V^{0}: V \in \mathscr{B}_{\tau}\right\}$. By Proposition 8 and the Banach-Alaoglu theorem, for each $V \in \mathscr{B}_{\tau}, V^{0}$ is convex, solid, and $\sigma\left(B(\Sigma)_{\tau}^{\prime}, B(\Sigma)\right)$-compact in $b a(\Sigma)$. It follows that $\tau \subset|\tau|(B(\Sigma)$, $\left.B(\Sigma)_{\tau}^{\prime}\right)$.

To show that $|\sigma|\left(B(\Sigma), B(\Sigma)_{\tau}^{\prime}\right) \subset \tau$, assume that $f_{\alpha} \rightarrow 0$ in $B(\Sigma)$ for $\tau$. Then, $\left|f_{\alpha}\right| \rightarrow 0$ for $\tau$ because $\tau$ is locally solid. Let $\mu \in b a(\Sigma)$ be such that $\Phi_{\mu} \in B(\Sigma)_{\tau}^{\prime}$. Then, $\left|\Phi_{\mu}\right| \in B(\Sigma)_{\tau}^{\prime}$ because $B(\Sigma)_{\tau}^{\prime}$ is an ideal of $B(\Sigma)^{*}$ (see Proposition 4). Hence, $\rho_{\mu}\left(f_{\alpha}\right)=\left|\Phi_{\mu}\right|\left(\left|f_{\alpha}\right|\right) \rightarrow 0$, and it follows that $f_{\alpha} \rightarrow 0$ for $|\sigma|\left(B(\Sigma), B(\Sigma)_{\tau}^{\prime}\right)$, as desired.

\section{The Mackey Topology $\tau(B(\Sigma), c a(\Sigma))$}

In this section we study the Mackey topology $\tau(B(\Sigma), c a(\Sigma))$ on $B(\Sigma)$. It is known that $(B(\Sigma), \tau(B(\Sigma), c a(\Sigma)))$ is a generalized DF-space, that is, $\tau(B(\Sigma), c a(\Sigma))$ is the finest locally convex topology agreeing with itself on norm-bounded sets in $B(\Sigma)$ (see [8, Corollary 11.8], [9, Section 4], [10, Theorem 2]).

Let $c a_{\mathbb{R}}(\Sigma)$ stand for the Dedekind complete Banach lattice of all countably additive measures $\mu: \Sigma \rightarrow \mathbb{R}$. Then, $c a_{\mathbb{R}}(\Sigma)$ is a band of $b a_{\mathbb{R}}(\Sigma)$. Moreover, the $\sigma$-order continuous dual $B_{\mathbb{R}}(\Sigma)_{c}^{*}$ of $B_{\mathbb{R}}(\Sigma)$ can be identified with $c a_{\mathbb{R}}(\Sigma)$ through the integration mapping $c a_{\mathbb{R}}(\Sigma) \ni \mu \mapsto \varphi_{\mu} \in B_{\mathbb{R}}(\Sigma)_{c}^{*}$, where $\varphi_{\mu}(u)=\int_{\Omega} u d \mu$ for all $u \in B_{\mathbb{R}}(\Sigma)$ (see [4, Theorem 13.5]).

Recall that a sequence $\left(u_{n}\right)$ in $B_{\mathbb{R}}(\Sigma)$ is said to be order convergent to $u \in B_{\mathbb{R}}(\Sigma)$ (in symbols, $u_{n} \stackrel{\text { (o) }}{\longrightarrow} u$ ) if there exists a sequence $\left(v_{n}\right)$ in $B_{\mathbb{R}}(\Sigma)^{+}$such that $\left|u_{n}-u\right| \leq v_{n} \downarrow 0$ (in the vector lattice sense; see [6, Definition 1.12]).

Definition 11. A linear functional $\Phi$ on $B(\Sigma)$ is said to be $\sigma$ order continuous if for each sequence $\left(f_{n}\right)$ in $B(\Sigma),\left|f_{n}\right| \stackrel{\text { (o) }}{\longrightarrow} 0$ in $B_{\mathbb{R}}(\Sigma)$ implies $\Phi\left(f_{n}\right) \rightarrow 0$.

Proposition 12. For $\mu \in b a(\Sigma)$ the following statements are equivalent:

(i) $\Phi_{\mu}$ is $\sigma$-order continuous.

(ii) $\Phi_{\mu}\left(f_{n}\right) \rightarrow 0$ for each uniformly bounded sequence $\left(f_{n}\right)$ in $B(\Sigma)$ such that $f_{n}(\omega) \rightarrow 0$ for all $\omega \in \Omega$.

(iii) $\Phi_{\mu}\left(u_{n}\right) \rightarrow 0$ for each sequence $\left(u_{n}\right)$ in $B(\Sigma)^{+}$such that $u_{n}(\omega) \downarrow 0$ for all $\omega \in \Omega$.

(iv) $\mu \in c a(\Sigma)$.

Proof. (i) $\Rightarrow$ (ii) Assume that $\Phi_{\mu}$ is $\sigma$-order continuous and let $f_{n}(\omega) \rightarrow 0$ for all $\omega \in \Omega$ and $\sup _{n}\left\|f_{n}\right\|<\infty$. Let $u_{n}(\omega)=\sup _{k \geq n}\left|f_{k}(\omega)\right|$ for $\omega \in \Omega, n \in \mathbb{N}$. Then, $u_{n} \in B_{\mathbb{R}}(\Sigma)$ and $\left|f_{n}(\omega)\right| \leq u_{n}(\omega) \downarrow 0$ for all $\omega \in \Omega$ and $n \in \mathbb{N}$. It follows that $\left|f_{n}\right| \stackrel{\text { (o) }}{\longrightarrow} 0$ in $B_{\mathbb{R}}(\Sigma)$, and hence, $\int_{\Omega} f_{n} d \mu \rightarrow 0$.

(ii) $\Rightarrow$ (iii) It is obvious.

(iii) $\Rightarrow$ (iv) Assume that (iii) holds, and let $A_{n} \downarrow \emptyset,\left(A_{n}\right) \subset$ $\Sigma$. Then, $\mathbb{1}_{A_{n}}(\omega) \downarrow 0$ for all $\omega \in \Omega$. Hence, $\mu\left(A_{n}\right)=$ $\int_{\Omega} \mathbb{1}_{A_{n}} d \mu \rightarrow 0$. (iv) $\Rightarrow$ (i) Assume that $\mu \in c a(\Sigma)$. Let $\left(f_{n}\right)$ be a sequence in $B(\Sigma)$ such that $\left|f_{n}\right| \stackrel{(o)}{\rightarrow} 0$ in $B_{\mathbb{R}}(\Sigma)$, that is, $\left|f_{n}\right| \leq u_{n} \downarrow 0$ for some sequence $\left(u_{n}\right)$ in $B(\Sigma)^{+}$. Then

$$
\left|\Phi_{\mu}\left(f_{n}\right)\right|=\left|\int_{\Omega} f_{n} d \mu\right| \leq \int_{\Omega}\left|f_{n}\right| d|\mu| \leq \int_{\Omega} u_{n} d|\mu|
$$

Note that $u_{n}(\omega) \downarrow 0$ for $|\mu|$-almost all $\omega \in \Omega$. Indeed, let $u_{n}(\omega) \downarrow u(\omega)$ for each $\omega \in \Omega$. Then, $u \in B_{\mathbb{R}}(\Sigma)$, so $A=\{\omega \in$ $\Omega: u(\omega)>0\} \in \Sigma$. If $\mu(A)>0$, then there exists $\varepsilon>0$ such that $A_{\varepsilon}=\{\omega \in \Omega: u(\omega) \geq \varepsilon\} \in \sum$ satisfies $|\mu|\left(A_{\varepsilon}\right)>0$. But then $u_{n} \geq \varepsilon \mathbb{1}_{A_{\varepsilon}}$ for each $n \in \mathbb{N}$, contradicting $u_{n} \downarrow 0$ in $B_{\mathbb{R}}(\Sigma)$. Hence, by Lebesgue Dominated Convergence theorem, we get $\int_{\Omega} u_{n} d|\mu| \rightarrow 0$, so $\int_{\Omega} f_{n} d \mu \rightarrow 0$.

By $B(\Sigma)_{c}^{*}$ we will denote the space of all $\sigma$-order continuous functionals on $B(\Sigma)$. For $\omega \in \Omega$ let $\delta_{\omega}$ denote the evaluation functional on $B(\Sigma)$, that is, $\delta_{\omega}(f)=f(\omega)$ for all $f \in$ $B(\Sigma)$. Then $\delta_{\omega} \in B(\Sigma)_{c}^{*}$ and it follows that $B(\Sigma)_{c}^{*}$ separates the points of $B(\Sigma)$.

From Proposition 12 and Lemma 5 if follows that for $\mu \epsilon$ $b a(\Sigma), \Phi_{\mu} \in B(\Sigma)_{c}^{*}$ if and only if $\left|\Phi_{\mu}\right| \in B(\Sigma)_{c}^{*}$. Since $c a(\Sigma)$ is an ideal of $b a(\Sigma)$, we see that $B(\Sigma)_{c}^{*}$ is an ideal of $B(\Sigma)^{*}$. For a subset $\mathscr{M}$ of $b a(\Sigma)$ let

$$
|\mathscr{M}|=\{|\mu|: \mu \in \mathscr{M}\} .
$$

Proposition 13. For a subset $\mathscr{M}$ of $c a(\Sigma)$ the following statements are equivalent:

(i) $\mathscr{M}$ is relatively $\sigma(c a(\Sigma), B(\Sigma))$-compact.

(ii) $\mathscr{M}$ is relatively $\sigma(c a(\Sigma), B(\Sigma))$-countably compact.

(iii) $\sup _{\mu \in \mathscr{M}}\|\mu\|<\infty$ and $\mathscr{M}$ is uniformly countably additive.

(iv) $\sup _{v \in \operatorname{conv}(\operatorname{sol}(\mathscr{M}))}\|\nu\|<\infty$ and $\operatorname{conv}(\operatorname{sol}(\mathscr{M}))$ is uniformly countably additive.

(v) $\operatorname{conv}(\operatorname{sol}(\mathscr{M}))$ is relatively weakly compact.

(vi) $\mathscr{M}$ is relatively weakly compact.

Proof. (i) $\Rightarrow$ (ii) It is obvious.

(ii) $\Rightarrow$ (iii) Assume that $\mathscr{M}$ is relatively $\sigma(c a(\Sigma), B(\Sigma))$ countably compact. It follows that $\mathscr{M}$ is $\sigma(c a(\Sigma), B(\Sigma))$ bounded, and since $\left\{\Phi_{\mu}: \mu \in \mathscr{M}\right\} \subset B(\Sigma)^{*}$, by the uniform boundedness theorem, $\sup _{\mu \in \mathscr{M}}\left\|\Phi_{\mu}\right\|<\infty$, that is, $\sup _{\mu \in \mathscr{M}}\|\mu\|<\infty$.

Assume, on the contrary, that $\mathscr{M}$ is not uniformly countably additive. Then there exist a sequence $\left(A_{n}\right)$ in $\Sigma$, a sequence $\left(\mu_{n}\right)$ in $\mathscr{M}$ and $\delta>0$ such that $A_{n} \downarrow \emptyset$ and $\left|\mu_{n}\left(A_{n}\right)\right| \geq \delta$ for all $n \in \mathbb{N}$. Let $\mathscr{C}=\left\{\mu_{n}: n \in \mathbb{N}\right\}$. Putting $\lambda=\sum_{n=1}^{\infty}\left(1 / 2^{n}\right)\left(\left|\mu_{n}\right| /\left(1+\| \mu_{n} \mid\right)\right)$, we see that $\lambda \in$ $\mathrm{ca}(\Sigma)^{+}$and $\mu_{n} \ll \lambda$ for all $n \in \mathbb{N}$. Hence, $\mathscr{C}$ is a relatively $\sigma\left(c a_{\lambda}(\Sigma), B(\Sigma)\right)$-countably compact subset of $c a_{\lambda}(\Sigma)$ (= the space of all $\lambda$-absolutely continuous measures in $c a(\Sigma))$. In view of the Radon-Nikodym theorem, for each $\mu \in c a_{\lambda}(\Sigma)$ there exists uniquely $f_{\mu} \in L^{1}(\lambda)$ such that $\mu(A)=\int_{A} f_{\mu} d \lambda$ for each $A \in \Sigma$. It follows that for every $g \in L^{\infty}(\lambda)$ we have $\int_{\Omega} f_{\mu} g d \lambda=\int_{\Omega} g d \mu$. Hence, the linear mapping $F_{\lambda}: c a_{\lambda}(\Sigma) \ni$ 
$\mu \mapsto f_{\mu} \in L^{1}(\lambda)$ is $\left(\sigma\left(c a_{\lambda}(\Sigma), B(\Sigma)\right), \sigma\left(L^{1}(\lambda), L^{\infty}(\lambda)\right)\right)$ continuous. Thus, the set $F_{\lambda}(\mathscr{C})=\left\{f_{\mu_{n}}: n \in \mathbb{N}\right\}$ is relatively $\sigma\left(L^{1}(\lambda), L^{\infty}(\lambda)\right)$-countably compact in $L^{1}(\lambda)$. Hence, by the Eberlein-Smulian theorem $\left\{f_{\mu_{n}}: n \in \mathbb{N}\right\}$ is relatively $\sigma\left(L^{1}(\lambda), L^{\infty}(\lambda)\right)$-compact in $L^{1}(\lambda)$. According to the Dunford-Pettis theorem (see [5, Chapter 3, Theorem 15]), the set $\left\{f_{\mu_{n}}: n \in \mathbb{N}\right\}$ in $L^{1}(\lambda)$ is uniformly integrable. Since $A_{n} \downarrow \emptyset$, we have that $\lambda\left(A_{n}\right) \rightarrow 0$, and hence $\mu\left(A_{n}\right)=$ $\int_{A_{n}} f_{\mu_{n}} d \lambda \rightarrow 0$, contradicting $\left|\mu_{n}\left(A_{n}\right)\right| \geq \delta$ for $n \in \mathbb{N}$.

(iii) $\Rightarrow$ (iv) Assume that $\sup _{\mu \in \mathscr{M}}\|\mu\|<\infty$ and $\mathscr{M}$ is uniformly countably additive. Then, $|\mathscr{M}|$ is uniformly countably additive (see [5, Chapter 1, Proposition 17]). Note that for $A \in \Sigma, \sup _{v \in \operatorname{sol}(\mathscr{M})}|\nu|(A)=\sup _{\mu \in \mathscr{M}}|\mu|(A)$. It follows that $\sup _{v \in \operatorname{sol}(\mathscr{M})}\|\nu\|<\infty$ and $\operatorname{sol}(\mathscr{M})$ is uniformly countably additive. Moreover, one can easily observe that for $A \in \Sigma, \sup _{v \in \operatorname{conv}(\operatorname{sol}(\mathscr{M}))}|\nu(A)|=\sup _{v \in \operatorname{sol}(\mathscr{M})}|\nu(A)|$ and $\sup _{\nu \in \operatorname{conv}(\operatorname{sol}(\mathscr{M}))}\|\nu\|=\sup _{\nu \in \operatorname{sol}(\mathscr{M})}\|\nu\|$. It follows that $\operatorname{conv}(\operatorname{sol}(\mathscr{M}))$ is uniformly bounded and uniformly countably additive.

(iv) $\Rightarrow$ (v) It is well known (see [11, Chapter 7, Theorem 13]).

$(\mathrm{v}) \Rightarrow(\mathrm{vi}) \Rightarrow$ (i) It is obvious.

Recall that a locally convex space $(L, \eta)$ is said to be strongly Mackey if every relatively $\sigma\left(L_{\eta}^{\prime}, L\right)$-countably compact set in $L_{\eta}^{\prime}$ is $\eta$-equicontinuous.

Corollary 14. The space $(B(\Sigma), \tau(B(\Sigma), c a(\Sigma)))$ is strongly Mackey.

Proof. Let $\mathscr{M}$ be a relatively $\sigma(c a(\Sigma), B(\Sigma))$-countably compact set in $c a(\Sigma)$. Then, by Proposition 13, the set $\mathrm{cl}_{\sigma}($ abs conv $\mathscr{M})$ is absolutely convex and $\sigma(c a(\Sigma), B(\Sigma))$ compact. Hence, ${ }^{0}\left(\mathrm{cl}_{\sigma}(\right.$ abs conv $\left.\mathscr{M})\right)$ is a $\tau(B(\Sigma), c a(\Sigma))$ neighborhood of 0 . It follows that $\mathscr{M}$ is $\tau(B(\Sigma), c a(\Sigma))$ equicontinuous.

Now we distinguish three natural classes of linear topologies on $B(\Sigma)$.

Definition 15. A locally solid topology $\tau$ on $B(\Sigma)$ is said to be

(i) $\sigma$-Lebesgue if $u_{n} \rightarrow 0$ for $\tau$ whenever $\left(u_{n}\right)$ is a sequence in $B(\Sigma)^{+}$such that $u_{n} \downarrow 0$ in $B_{\mathbb{R}}(\Sigma)$ (in the vector lattice sense).

(ii) a $\sigma$-smooth topology if $f_{n} \rightarrow 0$ for $\tau$ whenever $\left(f_{n}\right)$ is uniformly bounded sequence in $B(\Sigma)$ such that $f_{n}(\omega) \rightarrow 0$ for all $\omega \in \Omega$.

(iii) a $\sigma$-Dini topology if $u_{n} \rightarrow 0$ for $\tau$ whenever $\left(u_{n}\right)$ is a sequence in $B(\Sigma)^{+}$such that $u_{n}(\omega) \downarrow 0$ for all $\omega \in \Omega$.

Note that every $\sigma$-Lebesgue topology on $B(\Sigma)$ is $\sigma$ smooth, and every $\sigma$-smooth topology on $B(\Sigma)$ is $\sigma$-Dini.

Now, we characterize the Mackey topology $\tau(B(\Sigma), c a(\Sigma))$ on $B(\Sigma)$.

Theorem 16. (i) $\tau(B(\Sigma), c a(\Sigma))$ is a locally convex-solid topology. (ii) $\tau(B(\Sigma), c a(\Sigma))$ is the finest locally convex $\sigma$-Lebesgue topology on $B(\Sigma)$.

(iii) $\tau(B(\Sigma), c a(\Sigma))$ is the finest locally convex $\sigma$-smooth topology on $B(\Sigma)$.

(iv) $\tau(B(\Sigma), c a(\Sigma))$ is the finest locally convex $\sigma$-Dini topology on $B(\Sigma)$.

Proof. (i) Let $V$ be an absolutely convex and closed neighborhood of 0 for $\tau(B(\Sigma), c a(\Sigma))$. Then, the polar $V^{0}$ of $V$ (with respect to the solid dual system $\langle B(\Sigma), c a(\Sigma)\rangle$ ) is $\sigma(c a(\Sigma), B(\Sigma))$-compact. In view of Proposition 13 $\operatorname{conv}\left(\operatorname{sol}\left(V^{0}\right)\right)$ is relatively $\sigma(c a(\Sigma), B(\Sigma))$-compact and hence $\mathrm{cl}_{\sigma}\left(\operatorname{conv}\left(\operatorname{sol}\left(V^{0}\right)\right)\right)$ (the closure is taken in $\left.\sigma(c a(\Sigma), B(\Sigma))\right)$ is absolutely convex and $\sigma(c a(\Sigma), B(\Sigma))$-compact. Hence,

$$
{ }^{0}\left(\mathrm{cl}_{\sigma}\left(\operatorname{con} v\left(\operatorname{sol}\left(V^{0}\right)\right)\right)\right) \subset^{0}\left(\operatorname{sol}\left(V^{0}\right)\right) \subset^{0}\left(V^{0}\right)=V .
$$

It follows that ${ }^{0}\left(\operatorname{sol}\left(V^{0}\right)\right)$ is a convex and solid neighborhood of 0 for $\tau(B(\Sigma), c a(\Sigma)$ ) (see Proposition 8). This means that $\tau(B(\Sigma), c a(\Sigma))$ is a locally convex-solid topology on $B(\Sigma)$.

(ii) Now we shall show that $\tau(B(\Sigma), c a(\Sigma))$ is a $\sigma$ Lebesgue topology. Assume that $\left(u_{n}\right)$ is a sequence in $B(\Sigma)^{+}$ such that $u_{n} \quad \downarrow 0$ in the vector lattice $B_{\mathbb{R}}(\Sigma)$. Let $\mathscr{M}$ be a relatively $\sigma(c a(\Sigma), B(\Sigma))$-compact set in $c a(\Sigma)$. Then $\mathscr{M}$ is uniformly bounded and uniformly countably additive (see Proposition 13), and it follows that $|\mathscr{M}|$ is uniformly countably additive. Hence, by [10, Theorem 3] $|\mathscr{M}|$ is $\tau\left(B_{\mathbb{R}}(\Sigma), c a_{\mathbb{R}}(\Sigma)\right)$-equicontinuous. Hence, the polar ${ }_{\mathbb{R}}^{0}|\mathscr{M}|$ of $|\mathscr{M}|$ (with respect to the dual pair $\left\langle B_{\mathbb{R}}(\Sigma), c a_{\mathbb{R}}(\Sigma)\right\rangle$ ) is a $\tau\left(B_{\mathbb{R}}(\Sigma), c a_{\mathbb{R}}(\Sigma)\right.$-neighborhood of 0 in $B_{\mathbb{R}}(\Sigma)$. Let $\varepsilon>0$ be given. Since $\tau\left(B_{\mathbb{R}}(\Sigma), c a_{\mathbb{R}}(\Sigma)\right)$ is a $\sigma$-Lebesgue topology on $B_{\mathbb{R}}(\Sigma)$ (see [6, Example 18, page 178], [6, Corollary 6.29 and Theorem 3.20]), there exists $n_{\varepsilon} \in \mathbb{N}$ such that $\left|u_{n}\right| \in \varepsilon\left({ }_{\mathbb{R}}^{0}|\mathscr{M}|\right)$ for $n \geq n_{\varepsilon}$. Hence for $n \geq n_{\varepsilon}$ we get

$$
\begin{aligned}
p_{\mathscr{M}}\left(u_{n}\right) & =\sup \left\{\left|\int_{\Omega} u_{n} d \mu\right|: \mu \in \mathscr{M}\right\} \\
& \leq \sup \left\{\int_{\Omega} u_{n} d|\mu|: \mu \in \mathscr{M}\right\} \leq \varepsilon .
\end{aligned}
$$

It follows that $f_{n} \rightarrow 0$ for $\tau(B(\Sigma), c a(\Sigma))$, and this means that $\tau(B(\Sigma), c a(\Sigma))$ is a $\sigma$-Lebesgue topology on $B(\Sigma)$.

Now assume that $\tau$ is a locally convex-solid $\sigma$-Lebesgue topology on $B(\Sigma)$. Then $B(\Sigma)_{\tau}^{\prime} \subset B(\Sigma)_{c}^{*}$, and hence, $\tau \subset$ $\tau\left(B(\Sigma), B(\Sigma)_{\tau}^{\prime}\right) \subset \tau\left(B(\Sigma), B(\Sigma)_{c}^{*}\right)=\tau(B(\Sigma), c a(\Sigma))$.

(iii) From (ii) it follows that $\tau(B(\Sigma), c a(\Sigma))$ is a $\sigma$-smooth topology.

Now let $\tau$ be a locally convex $\sigma$-smooth topology on $B(\Sigma)$. Then by Proposition 12 we get $B(\Sigma)_{\tau}^{\prime} \subset B(\Sigma)_{c}^{*}$. Hence, $\tau \subset$ $\tau\left(B(\Sigma), B(\Sigma)_{\tau}^{\prime}\right) \subset \tau\left(B(\Sigma), B(\Sigma)_{c}^{*}\right)=\tau(B(\Sigma), c a(\Sigma))$.

(iv) Clearly $\tau(B(\Sigma), c a(\Sigma))$ is a $\sigma$-Dini topology on $B(\Sigma)$. Assume that $\tau$ is a locally convex $\sigma$-Dini topology on $B(\Sigma)$. In view of Proposition 12, $B(\Sigma)_{\tau}^{\prime} \subset B(\Sigma)_{c}^{*}$ and it follows that $\tau \subset \tau(B(\Sigma), c a(\Sigma))$.

A characterization of relative weak compactness in the Banach space $c a(\Sigma)$ in terms of uniform boundedness and 
uniform countable additivity of measures belongs to the classical results in measure theory (see [11, Chapter 7, Theorem 13], [8, Theorem 11.6], [12, Theorem 1.1]). Now, using the properties of the Mackey topology $\tau(B(\Sigma), c a(\Sigma))$ we are ready to add Grothendieck type conditions to this characterization.

Corollary 17. For a subset $\mathscr{M}$ of $c a(\Sigma)$ the following statements are equivalent:

(i) $\mathscr{M}$ is a relatively weakly compact.

(ii) $\sup _{\mu \in M}\|\mu\|<\infty$ and $\int_{\Omega} f_{n} d \mu \rightarrow 0$ uniformly for $\mu \epsilon$ $M$ whenever $\left(f_{n}\right)$ is a uniformly bounded sequence in $B(\Sigma)$ such that $f_{n}(\omega) \rightarrow 0$ for all $\omega \in \Omega$.

(iii) $\sup _{\mu \in M}\|\mu\|<\infty$ and $\int_{\Omega} u_{n} d \mu \rightarrow 0$ uniformly for $\mu \in \mathscr{M}$ whenever $\left(u_{n}\right)$ is a sequence in $B(\Sigma)^{+}$such that $u_{n}(\omega) \downarrow 0$ for all $\omega \in \Omega$.

Proof. (i) $\Rightarrow$ (ii) Assume that $\mathscr{M}$ is relatively weakly compact. Then, in view of Corollary $14 \mathscr{M}$ is bounded and $\tau(B(\Sigma), c a(\Sigma))$-equicontinuous. Let $\left(f_{n}\right)$ be a uniformly bounded sequence in $B(\Sigma)$ such that $f_{n}(\omega) \rightarrow 0$ for all $\omega \in \Omega$. Since $\tau(B(\Sigma), c a(\Sigma))$ is a $\sigma$-smooth topology on $B(\Sigma)$ (see Theorem 16), we obtain that $f_{n} \rightarrow 0$ for $\tau(B(\Sigma), c a(\Sigma)$ ). Now let $\varepsilon>0$ be given. Then, there exists a $\tau(B(\Sigma), c a(\Sigma))$ neighborhood $V$ of 0 such that $\sup _{\mu \in \mathscr{M}}\left|\int_{\Omega} f d \mu\right| \leq \varepsilon$ for all $f \in V$. Hence, there exists $n_{\varepsilon} \in \mathbb{N}$ such that $f_{n} \in V$ for $n \geq n_{\varepsilon}$, and it follows that $\sup _{\mu \in \mathscr{M}}\left|\int_{\Omega} f_{n} d \mu\right| \leq \varepsilon$ for $n \geq n_{\varepsilon}$, as desired.

(ii) $\Rightarrow$ (iii) It is obvious.

(iii) $\Rightarrow$ (i) Assume that (iv) holds and let $A_{n} \downarrow \emptyset,\left(A_{n}\right) \subset \Sigma$. Then, $\mathbb{1}_{A_{n}}(\omega) \downarrow 0$ for all $\omega \in \Omega$. Hence $\sup _{\mu \in M}\left|\mu\left(A_{n}\right)\right|=$ $\sup _{\mu \in \mathscr{M}}\left|\int_{\Omega}^{n} \mathbb{1}_{A_{n}} d \mu\right| \rightarrow 0$, that is, $\mathscr{M}$ is uniformly countably additive. Hence, $\mathscr{M}$ is relatively weakly compact (see [11, Chapter 7, Theorem 13]).

Remark 18. One can observe that Corollary 17 is closely related to a Grothendieck's characterization of relative weak compactness in the space of bounded complex Radon measures on a locally compact space (see [13, Theorem 2]).

Now we define a class of linear operators on $B(\Sigma)$.

Definition 19. Let $(E, \xi)$ be a locally convex Hausdorff space (briefly, lcHs). A linear operator $T: B(\Sigma) \rightarrow E$ is said to be $\sigma$ order continuous if for each sequence $\left(f_{n}\right)$ in $B(\Sigma),\left|f_{n}\right| \stackrel{(\text { o) }}{\longrightarrow} 0$ in $B_{\mathbb{R}}(\Sigma)$ implies $T\left(f_{n}\right) \rightarrow 0$ for $\xi$.

For terminology and basic results concerning the integration with respect to vector measures, we refer the reader to [14-17].

Let $(E, \xi)$ be a quasicomplete lcHs. Let $m: \Sigma \rightarrow E$ be a $\xi$-bounded measure (i.e., the range of $m$ is $\xi$-bounded in $E)$. Given $f \in B(\Sigma)$, let $\left(s_{n}\right)$ be a sequence of $\Sigma$-simple scalar functions that converges uniformly to $f$ on $\Omega$. Following [14, Definition 1] we say that $f$ is $m$-integrable and define

$$
\int_{\Omega} f d m:=\xi-\lim \int_{\Omega} s_{n} d m
$$

The $\int_{\Omega} f d m$ is well defined (see [14, Lemma 5]) and the map $T_{m}: B(\Sigma) \rightarrow E$ given by $T_{m}(f)=\int_{\Omega} f d m$ is $(\|\cdot\|, \xi)$-continuous and linear, and for each $e^{\prime} \in E_{\xi}^{\prime}$

$$
e^{\prime}\left(\int_{\Omega} f d m\right)=\int_{\Omega} f d\left(e^{\prime} \circ m\right) \text { for } f \in B(\Sigma)
$$

(see [14, Lemma 5]). Conversely, let $T: B(\Sigma) \rightarrow E$ be a $(\| \cdot$ $\|, \xi)$-continuous linear operator, and let $m(A)=T\left(\mathbb{1}_{A}\right)$ for $A \in \Sigma$. Then, $m: \Sigma \rightarrow E$ is a $\xi$-bounded vector measure, called the representing measure of $T$ and $T_{m}(f)=T(f)$ for $f \in B(\Sigma)$ (see [14, Definition 2]).

Recall that a measure $m: \Sigma \rightarrow E$ is said to be $\xi$-countably additive if $m\left(A_{n}\right) \rightarrow 0$ for $\xi$ whenever $A_{n} \downarrow \emptyset,\left(A_{n}\right) \subset \Sigma$.

The following characterization of $\sigma$-order continuous operators from $B(\Sigma)$ into a quasicomplete lcHs $(E, \xi)$ displays the close connection between the Mackey topology $\tau(B(\Sigma), c a(\Sigma))$ on $B(\Sigma)$ and $E$-valued $\xi$-countably additive measures.

Proposition 20. Assume that $(E, \xi)$ is a quasicomplete $l c H s$. Then for a $\xi$-bounded measure $m: \Sigma \rightarrow E$ the following statements are equivalent:

(i) $T_{m}$ is $\left(\tau(B(\Sigma), c a(\Sigma)), \tau\left(E, E_{\xi}^{\prime}\right)\right)$-continuous.

(ii) $T_{m}$ is $(\tau(B(\Sigma), c a(\Sigma)), \xi)$-continuous.

(iii) $T_{m}$ is sequentially $(\tau(B(\Sigma), c a(\Sigma)), \xi)$-continuous.

(iv) $T_{m}$ is $\sigma$-order continuous.

(v) $T_{m}\left(f_{n}\right) \rightarrow 0$ for $\xi$ whenever $\left(f_{n}\right)$ is a uniformly bounded sequence in $B(\Sigma)$ such that $f_{n}(\omega) \rightarrow 0$ for all $\omega \in \Omega$.

(vi) $T_{m}\left(u_{n}\right) \rightarrow 0$ for $\xi$ for each sequence $\left(u_{n}\right)$ in $B(\Sigma)^{+}$such that $u_{n}(\omega) \rightarrow 0$ for all $\omega \in \Omega$.

(vii) $m$ is $\xi$-countably additive.

Proof. (i) $\Rightarrow$ (ii) $\Rightarrow$ (iii) It is obvious.

(iii) $\Rightarrow$ (iv) Assume that $T_{m}$ is sequentially $(\tau(B(\Sigma)$, $c a(\Sigma)), \xi)$-continuous, and let $\left(f_{n}\right)$ be a sequence in $B(\Sigma)$ such that $\left|f_{n}\right| \stackrel{\text { (o) }}{\longrightarrow} 0$ in $B_{\mathbb{R}}(\Sigma)$. Then, by Theorem 16, $f_{n} \rightarrow 0$ for $\tau(B(\Sigma), c a(\Sigma))$. Hence, $T_{m}\left(f_{n}\right) \rightarrow 0$ for $\xi$, that is, $T$ is $\sigma$-order continuous.

(iv) $\Rightarrow$ (v) Assume that $T_{m}$ is $\sigma$-order continuous, and assume that $\left(f_{n}\right)$ is a uniformly bounded sequence in $B(\Sigma)$ such that $f_{n}(\omega) \rightarrow 0$ for all $\omega \in \Omega$. Then $\left|f_{n}\right| \stackrel{\text { (o) }}{\longrightarrow} 0$ in $B_{\mathbb{R}}(\Sigma)$ (see the proof of Proposition 12). Hence, $T_{m}\left(f_{n}\right) \rightarrow 0$ for $\xi$, as desired.

(v) $\Rightarrow$ (vi) It is obvious.

(vi) $\Rightarrow$ (vii) Assume that (vi) holds, and let $A_{n} \downarrow \emptyset,\left(A_{n}\right) \subset$ $\Sigma$. Then, $\mathbb{1}_{A_{n}}(\omega) \downarrow 0$ for all $\omega \in \Omega$, and it follows that $\mu\left(A_{n}\right)=T_{m}\left(\mathbb{1}_{A_{n}}\right) \rightarrow 0$ for $\xi$. This means that $m$ is $\xi$ countably additive.

(vii) $\Rightarrow$ (i) Assume that $m$ is $\xi$-countably additive. Then $e^{\prime} \circ m \in c a(\Sigma)$ for each $e^{\prime} \in E_{\xi}^{\prime}$, and in view of (28) and Proposition 12 we get $e^{\prime} \circ T_{m} \in B(\Sigma)_{c}^{*}$ for each $e^{\prime} \in E_{\xi}^{\prime}$. Hence, $T_{m}$ is $\left(\sigma\left(B(\Sigma), B(\Sigma)_{c}^{*}\right), \sigma\left(E, E_{\xi}^{\prime}\right)\right)$-continuous (see [7, Theorem 9.26]). It follows that $T_{m}$ is $\left(\tau\left(B(\Sigma), B(\Sigma)_{c}^{*}\right), \tau\left(E, E_{\xi}^{\prime}\right)\right)$ continuous (see [7, Example 11, page 149]). 


\section{Acknowledgments}

The author wishes to thank the referee for useful suggestions that have improved the paper.

\section{References}

[1] A. C. Zaanen, Riesz Spaces II, vol. 30 of North-Holland Mathematical Library, North-Holland, Amsterdam, The Netherlands, 1983.

[2] T. W. Ma, Banach-Hilbert Spaces, Vector Measures and Group Representations, World Scientific, River Edge, NJ, USA, 2002.

[3] H. H. Schaefer, Banach Lattices and Positive Operators, Springer, New York, NY, USA, 1974.

[4] C. D. Aliprantis and K. C. Border, Infinite-Dimensional Analysis, Springer, Berlin, Germany, 2nd edition, 1999.

[5] J. Diestel and J. J. Uhl Jr., Vector Measures, Mathematical Surveys, no. 15, American Mathematical Society, Providence, RI, USA, 1977.

[6] C. D. Aliprantis and O. Burkinshaw, Locally solid Riesz Spaces with Applications to Economics, vol. 105 of Mathematical Surveys and Monographs, American Mathematical Society, Providence, RI, USA, 2nd edition, 2002.

[7] C. D. Aliprantis and O. Burkinshaw, Positive Operators, vol. 119 of Pure and Applied Mathematics, Academic Press, New York, NY, USA, 1985.

[8] W. H. Graves, On the Theory of Vector Measures, vol. 195 of Memoirs of the American Mathematical Society, American Mathematical Society, Providence, RI, USA, 1977.

[9] W. H. Graves and W. Ruess, "Compactness in spaces of vectorvalued measures and a natural Mackey topology for spaces of bounded measurable functions," Contemporary Mathematics, vol. 2, pp. 189-203, 1980.

[10] S. S. Khurana, "A topology associated with vector measures," The Journal of the Indian Mathematical Society, vol. 45, no. 1-4, pp. 167-179, 1981.

[11] J. Diestel, Sequences and Series in Banach Spaces, vol. 92 of Graduate Texts in Mathematics, Springer, New York, NY, USA, 1984.

[12] X. D. Zhang, "On weak compactness in spaces of measures," Journal of Functional Analysis, vol. 143, no. 1, pp. 1-9, 1997.

[13] A. Grothendieck, "Sur les applications linéaires faiblement compactes d'espaces du type $C(K)$," Canadian Journal of Mathematics, vol. 5, pp. 129-173, 1953.

[14] T. V. Panchapagesan, "Applications of a theorem of Grothendieck to vector measures," Journal of Mathematical Analysis and Applications, vol. 214, no. 1, pp. 89-101, 1997.

[15] J. Hoffmann-Jørgensen, "Vector measures," Mathematica Scandinavica, vol. 28, pp. 5-32, 1971.

[16] D. R. Lewis, "Integration with respect to vector measures," Pacific Journal of Mathematics, vol. 33, pp. 157-165, 1970.

[17] T. V. Panchapagesan, "Characterizations of weakly compact operators on $C_{0}(T)$," Transactions of the American Mathematical Society, vol. 350, no. 12, pp. 4849-4867, 1998. 


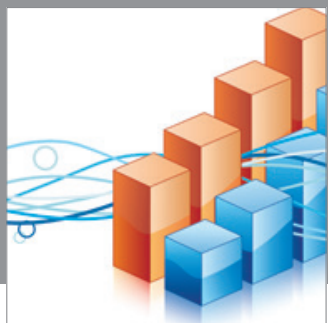

Advances in

Operations Research

mansans

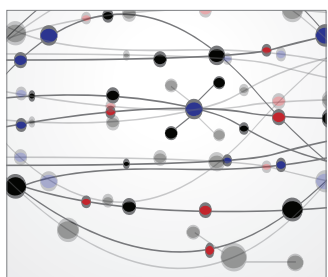

The Scientific World Journal
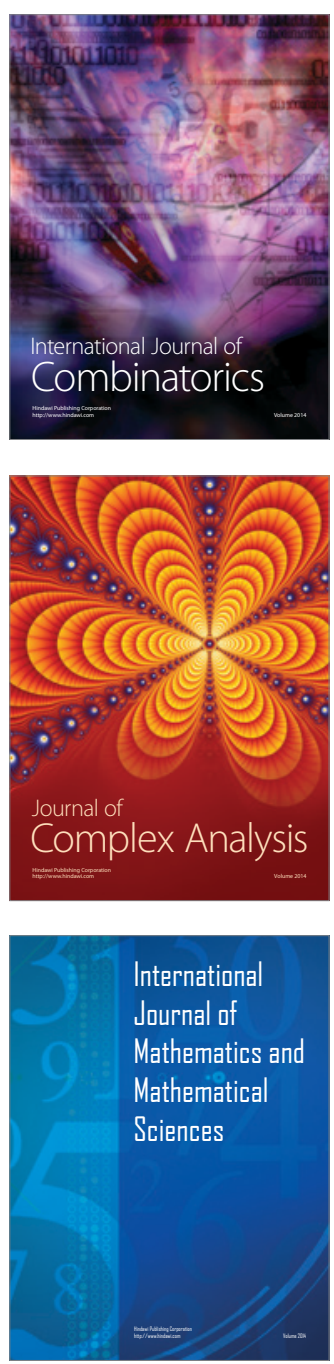
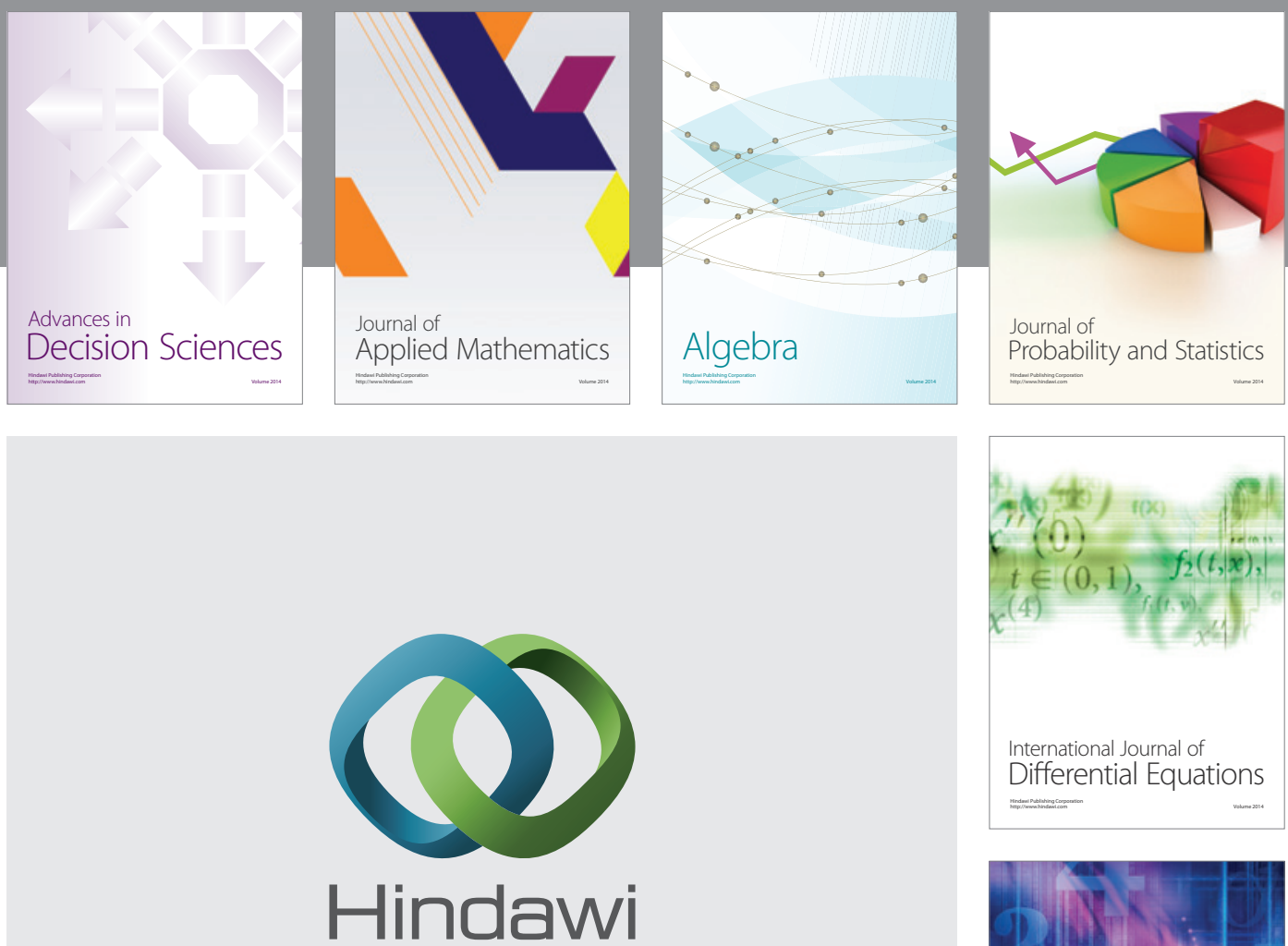

Submit your manuscripts at http://www.hindawi.com
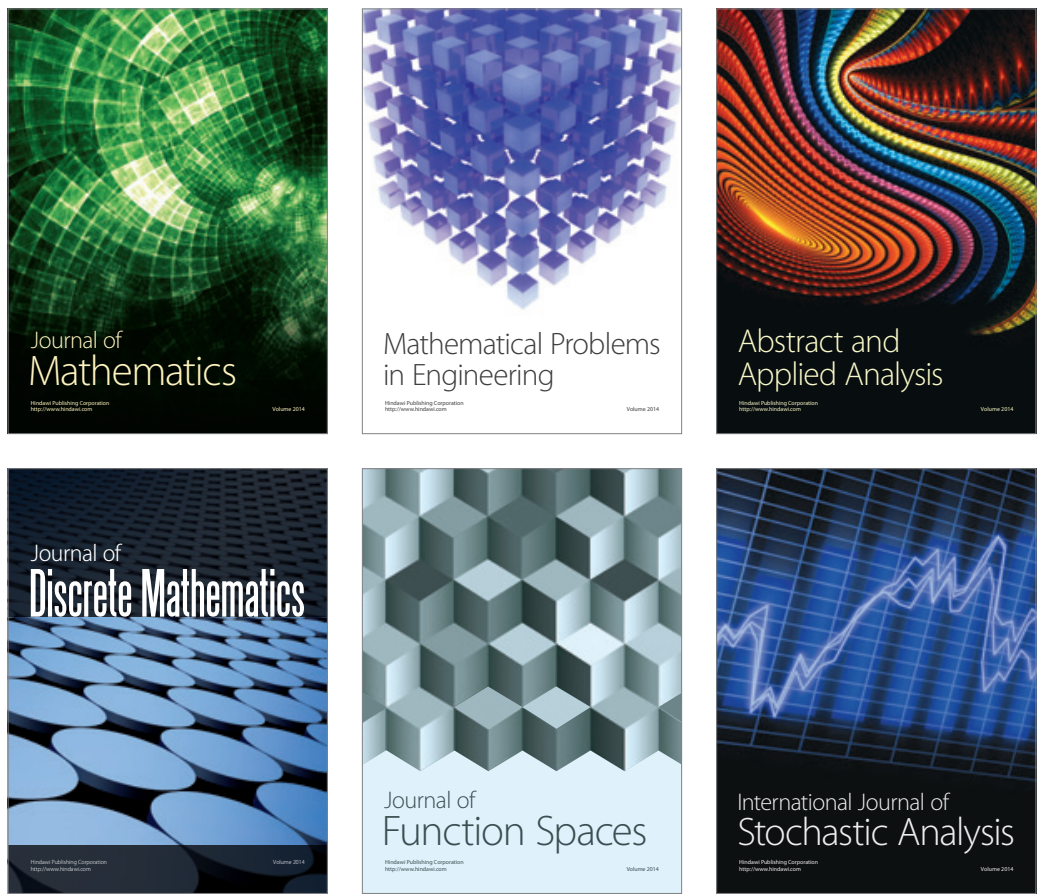

Journal of

Function Spaces

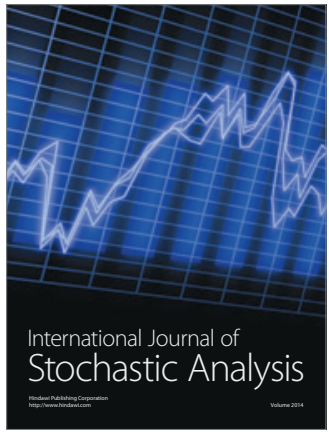

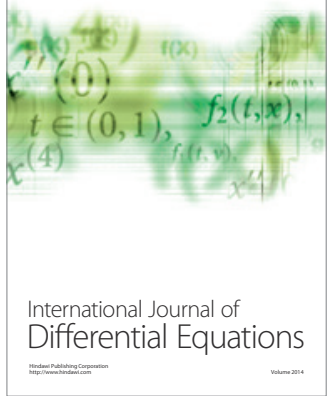
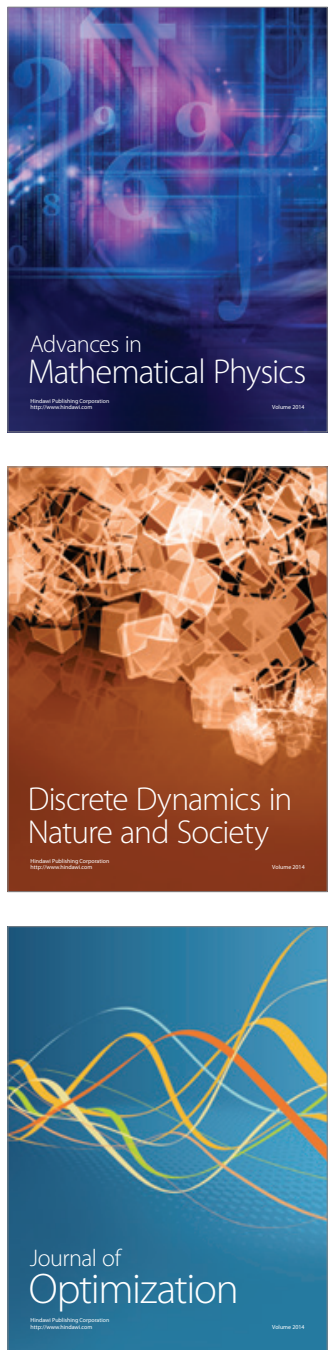